\title{
I mplementation and evaluation of a mental health program for Ni Vanuatu nurses
}

\author{
S. Webster, J. Allnutt, P. Boss \\ School of Nursing, Midwifery and Paramedicine, Australian Catholic University, North Sydney, Australia. \\ Correspondence: Sayumporn Webster. Address: School of Nursing, Midwifery and Paramedicine, Australian Catholic \\ University, 40 Edward Street North Sydney NSW 2060, PO Box 968, North Sydney, Australia. \\ Email: Sue.Webster@acu.edu.au
}

Received: January 24, $2013 \quad$ Accepted: July 2, $2013 \quad$ Online Published: August 15, 2013

DOI : 10.5430/jnep.v3n12p177 URL: http://dx.doi.org/10.5430/jnep.v3n12p177

\section{Abstract}

Objective: This is a case study, examined in order to understand the issues associated with implementing a mental health nursing program in Vanuatu. The participant was a registered nurse identified as leader in nursing practice, with advanced knowledge and skills in mental health for his role in Vanuatu. This paper also explores the perceptions and experiences of the participant as part of his preparation for mental health education.

Method: Using a case study design, quantitative data was collected using semi-structured interviews and researcher journaling. Data were analysed using an inductive thematically approach. Mixed methods and concurrent data analysis contributed to the rigor of this study.

Results: A Ni Vanuatu nurse (Fellow) was sponsored by the Australian Government Overseas Aid Program (AusAID) Fellowship to participate in a short-term, non-award study program of mental health theory and practice over 18 weeks in the Australian setting. The program enabled him to join 2nd year undergraduate nursing students undertaking the core Mental Health Unit at ACU. The findings indicate that the Fellow valued the opportunity to practice mental health nursing activities in a supervised and mentored environment that promoted active learning for developing clinical competence and increased confidence in mental health practice. Of particular value was the ability to undertake a range of clinical attachment opportunities that would not have been possible in Vanuatu.

Conclusion: Vanuatu has a significant and identified deficit in mental health services, with little funding, infrastructure, resources or staffing allocated. The relevance of the introduction of this program, in a country where nursing education still takes place at certificate level, where there was little understanding of mental health conditions and interventions and where there were no real opportunities for clinical attachment or mentorship, cannot be understated.

\section{Key words}

Mental health nursing, Education, Program implementation, Developing country

\section{Introduction}

Vanuatu has been working for several years to develop a baseline infrastructure and workforce to address the growing need for mental health services. In 2009, a team from Australian Catholic University (ACU), North Sydney Campus, was successful in obtaining an Australian Leadership Awards (ALA) Fellowship, through AusAID. The Fellowship targeted 
education in mental health, a priority health development area identified by the Vanuatu Ministry of Health ${ }^{[1]}$, initially bringing one nurse with an expressed and demonstrated interest in mental health to complete a program taught in Australia.

\subsection{Background of the study}

The Nursing program in Vanuatu contained limited mental health theory and really no relevant clinical attachment. There was and continues to be no postgraduate mental health nursing course in Vanuatu and no budget to establish formal staff training in this field. The Fellowship program was initiated at a time when the VMoH was looking for partnerships to assist them to develop their mental health services ${ }^{[2]}$.

Demographically, Vanuatu is an island nation situated in the South Pacific. It is an archipelago consisting of more than 80 islands, of which approximately two thirds are inhabited. The population of approximately $245,000^{[3]}$ people is distributed across an area spanning some 12,200 square kilometers and it is estimated that seventy-seven percent of the population live in rural areas with a subsistence lifestyle based on farming and fishing ${ }^{[3]}$. There are five hospitals, with the teaching and referral hospital being located in Port Vila, the capital. The hospitals are supported by twenty five health centres that provide integrated care, staffed by registered nurses and 123 health aid posts ${ }^{[4]}$, generally staffed by village health workers.

In 2005, the World Health Organization (WHO) ${ }^{[5,6]}$ undertook an analysis of mental health needs and resources in Pacific Island countries. The analysis highlighted that most Pacific nations had growing rates of mental health problems; however the services and resources to help people were generally not available. However some South Pacific island countries, such as Fiji were successful in developing strong education programs and comprehensive mental health services. In some South Pacific island countries, mental health care equates with putting people in jails, rather than treating and supporting their mental health well-being ${ }^{[7]}$. The Pacific Islands Mental Health Network (PIMHnet) (2009) ${ }^{[8,9]}$ reported that it was common for South Pacific island countries to have no mental health policy, strategic plan, budget, or legislation to protect the rights of people with mental health issues and few (if any) mental health related skills in their workforce. PIMHnet, a WHO initiative funded by NZAid ${ }^{[10,11]}$, aims to build and mentor a mental health workforce, reducing stigma in the community, providing limited training for the informal mental health workforce, and advocating with Government and NGOs. Again, countries such as Fiji have worked to overcome many of these barriers to develop sustainable mental health services ${ }^{[12,13]}$.

\subsection{Mental health service development in Vanuatu}

Mental health in Vanuatu has struggled to be recognized at all levels of society, with small incremental change being noted in recent years. Despite this, basic mental health services are still unavailable in most of the islands, leaving people to rely on traditional or religious approaches to mental health care at the village level ${ }^{[7,8]}$. In Vanuatu, pastors and church leaders are well respected members of the community. The Church is often the first point of call for many people with mental health issues, or their families. In the community, prejudices frequently exist about mental health issues where it is believed that mental illness reflects a weak faith or possession by demons. However, in recent years there has been an increasing interest among churches to improve their understanding of mental health and its treatment. Kaloris ${ }^{[8]}$ stressed the need to engage pastors and church leaders in local service planning due to the integral and influential role they play in community.

In 2009, when the Fellowship was established, mental health services in Vanuatu were limited to a total of four (4) designated beds with lock-down capacity (two each at Vila Central Hospital and Luganville Hospital, Santo Espiritu) where the care of psychiatric patients was provided by generalist staff as there were no psychiatric or qualified mental health nurse workforce.

Concurrent with the Fellowship being undertaken in Australia, the VMOH was working on policies to address the growing issues associated with mental health. Two landmark documents were released by the VMoH, the Vanuatu Mental Health 
Policy and Strategic Plan 2009-2015 ${ }^{[1,4]}$ and a National Mental Health Training Plan (2009-2014) ${ }^{[4]}$. The VMoH declared that their priority was to strengthen and build capacity within the existing health professionals to further improve mental health services within the primary health care system. The Fellowship program seemed well aligned with the VMoH approach to workforce development.

\subsection{The Australian Leadership Awards (ALA) program}

In 2009 the $\mathrm{VMoH}$ was beginning to recognize the need to prioritise mental health in the health agenda, and was developing partnerships to assist them in the establishment of mental health services ${ }^{[18]}$. One strategy was through collaboration with the ACU, North Sydney Campus to successfully obtain an Australian Leadership Awards (ALA) Fellowship, through AusAID. This Fellowship targeted education in mental health, a priority health development area for Vanuatu, by establishing an under-graduate mental health training position in Australia for registered nurses from Vanuatu.

\section{Purpose of the study}

The objective of this case study is to provide deeper understanding and explanations to the issues associated with implementing a mental health nursing program in Vanuatu. The participant account and insights into causal relationship issues:

- Describe one approach that complemented a range of strategies endorsed by the $\mathrm{VMoH}$.

- Investigate the perceptions and experiences of the participant as part of his preparation for mental health education.

\section{Subject and method}

\subsection{Study design}

\section{Method}

A case study, using a mixed method approach, was used to explore the Fellow's experiences with the program. A case study approach was chosen because it parallels real-life situations and was able to describe the phenomenon of the participant's experience of studying in a foreign country and then how he applied his mental health knowledge upon return to his home context. Geertz ${ }^{[13]}$ stated that in most in-depth case studies "The Field" itself is a "powerful disciplinary force: assertive, demanding, even coercive” (p. 119). Case studies of an individual in health care research often involve in-depth interviews with the participant and key informant and research journaling ${ }^{[14]}$.

\section{Recruitment (Participant)}

The Vanuatu Ministry of Health undertook selection of a suitable participant against selection criteria. There was a request from the academic partner that the nominated candidate had demonstrated some degree of interest and leadership with respect to mental health care. The Fellow selected by the Ministry of Health was a registered nurse with approximately seven years experience and an expressed interest in mental health and was an active member of the Pacific Island Mental Health Network (PIMHnet).

\section{The Fellowship program}

The Fellowship program was designed to provide the Ni Vanuatu registered nurse with an understanding of mental health issues through participation in a short-term program of mental health theory and clinical attachment in the Australian setting. The short-term non award study program took place over 18 weeks and included both theory and practical 
components. The Fellow joined 2nd year undergraduate nursing students undertaking the core Mental Health Unit at ACU. The Fellow completed 5 hours of face-to-face learning per week, supplemented with 6 weeks of clinical attachments across a range of mental health settings, including affiliated Catholic Hospital settings, the NSW Ambulance Training Unit, a rural mental health inpatient unit, Aboriginal Medical Services and other non-government mental health services.

The mental health theoretical component provided the Fellow with basic foundations in the areas of assessment, diagnostic skills, care planning and management of patients. The clinical attachments ensured that the Fellow gained exposure to practical nursing environments offered in both public and private facilities, in community and inpatient settings where he could apply and practice his newly acquired skills and knowledge, under supervision. In addition, relevant mental health workshops to assist the Fellow gain insight into a variety of professional issues associated with policy development and clinical practice were offered. Completion of the Fellowship Program was contingent upon the successful completion of clinical attachments and a written assignment. The program encouraged the Fellow to consider issues in a cultural context to assist him to develop an understanding of the factors influencing mental health and illness, and how they might present in Vanuatu.

\subsection{Data collection}

Following ethics approval and consent, data was collected from in-depth interviews. The data was thematically analysed using content analysis.

The project team was familiar with Vanuatu's health system and culture, having participated in a cultural immersion program with the ACU undergraduate nursing programme over several years, and so were able to explore the differences in culture with the Fellow and give support where required. Prior to the Fellow's arrival in Sydney a needs analysis ${ }^{[18]}$ was undertaken to assess his learning requirements both in Australia and also in relation to $\mathrm{VMoH}$ expectations on his return at the end of the course.

Following the arrival of the Fellow in Sydney, members of the project team assisted him to acclimatize to his new environment and adapt to the cultural changes. This included a tour of the University campus, provision of warm clothes (as it was mid-winter), exploration of transportation services, and some social activities. Rose \& Best ${ }^{[15,16]}$ described the importance of orientation programs for explaining professional behaviours, social interactions and ways of addressing educators. The Fellow had to adjust to the expectations of academia as well as the clinical context. Issues such as punctuality were emphasised as punctuality is of less importance in the Vanuatu culture. To obtain information and gain an insight into the experiences of the Fellow a post program interview was conducted. Prior to conducting the interview the Fellow signed a consent form allowing the interview to be recorded.

\subsection{Post program interview}

The Fellow's experiences of the program and its effectiveness were explored using an in-depth semi structured interview. The interview used open ended questions, for example "could you tell me about your experiences in Australia?" and "Can we explore your view on...” to discover the meaning of the lived experience as an international student living and studying in a foreign country.

In the interview, the interviewer also captured conversations and non-verbal responses through note taking to add richness to the data ${ }^{[14]}$. The interview and the interviewer notes were transcribed verbatim by one of the authors.

\subsection{Data analysis}

Two authors skilled in qualitative methods individually immersed themselves in the transcriptions using the computer software program NVivo 9.2 to manage the data. Thematic analysis was performed to create and establish meaningful patterns from the transcripts and from this, codes categories and concepts were refined into four main themes ${ }^{[15]}$ (see Table 1). 
Table 1. Theme and Categories

\begin{tabular}{ll}
\hline Categories & Successful Scholarship \\
\hline \multirow{3}{*}{ Immigration } & Influencing factors \\
& Unfamiliar procedure \\
& Difficulty with process \\
& Clarifying cost \\
Belonging and family & Time wasting \\
& Anxiety, fear of new environment \\
Integration and support & Missing family \\
& Loneliness \\
Living and studying in Australia. & Help from the team \\
& Found a friend \\
& Literacy \\
& Confidence \\
\end{tabular}

\section{Results}

The Fellow faced a number of challenges both in his preparation to undertake the Fellowship and during his time in Australia. The four main themes to be discussed here are: immigration; belonging and family; integration and support; and living and studying in Australia.

\section{Themes}

\section{I mmigration}

Obtaining a Visa was identified as a particularly difficult and very stressful issue for the Fellow. He was required to submit a range of documents in order to process the visa application. However, for a variety of reasons these documents were hard to find and at times even harder to negotiate copies of.

"When I went to apply the visa I was asked for documents like my birth certification and marriage license which I did not have. I spent many days trying to get copies of these documents... I spent a lot of time waiting in government offices... I had no idea that it took so long... maybe two months or more to get the visa organized... then my application have to go to Fiji for processing. It was good when Mrs J finally came to Vila as she helped me really a lot”.

The upfront costs were a burden with the cost of applying for a Visa, medical examination and immunization requirements being very expensive, requiring reliance on family members for financial assistance, although he was later able to recoup the costs following arrival in Australia. He commented:

"It was hard for me to find the money to come to Australia. I have no idea the visa, medical assessment, chest $\mathrm{X}$-ray and immunisation cost so much. I had to wait two weeks for one vaccine because there was none available. I had to go to the private hospital to have my tests so I had to pay at the time I have the examination. I asked for help from the Ministry but they said they could not help... same for the Uni. I had to ask my family if they could loan me some money".

\section{Belonging and family}

Funding enabled only for the Fellow to come to Australia. He had to leave his family at home and he struggled with his emotions especially being absent for the birth of his second child. At times he felt vulnerable, isolated, and lonely. With respect to socialisation the Fellow commented: 
"I was a very lonely, isolated especially at the beginning and found it very stressful. I am used to having many members of my family with me... 14 people live with me in my house. I didn't make friends at Uni... there were different people in my classes each week but I had a few male friends at the hostel where I stayed. Next time it would be better if two students came together".

Whilst describing his loneliness, he spoke about the mobile phone as a lifeline to ring home:

"Sometimes I rang home four times a day to speak to my family... I got upset as the mobile phone ran out of credit all the time. It cost so much to ring home but I needed to speak to my wife and family especially at night when I was by myself. It’s a long time to be away from my family”.

\section{I ntegration and support}

The Fellow found the support from the team was well organised and helpful.

"You all gave wonderful support to me, especially in those first few weeks. You all were very helpful... in Vanuatu with getting the Visa and medical stuff and in settling me in... and setting up my mobile, and a bank account banking... helped me socially and showed me around a lot. ... and in my first week fear of transportation, especially the railway station. It took me a month to get used to the transport system”.

There was no train transport in Vanuatu and the Fellow found the prospect of catching the train (iron horse) very daunting even though he was excited for the experience at the same time.

\section{Living and study in Australia}

The Fellow highlighted a wide range of issues both positive and negative about his experiences, especially of academic work. He identified that he had to quickly learn academic survival skills including the need to learn and develop notetaking skills, the level of literacy expected for assignment writing and participation in self-directed learning outside the classroom. Another challenge highlighted by the Fellow was his lack of confidence and competence with accessing on-line resources to support his studies, and the use of the computer to complete work. In the post-interview the Fellow identified,

“... Still need a lot of help from the library. My first time experience. ... I think I will be able to search on the internet in Vanuatu... the study was stressful, I was not prepared... I struggle to cope. I knew it would be a challenge... The Uni needs to prepare us better ... academically, especially for writing...; I learnt from my mentor... about my assessment,... I did not do well... I felt bad about it but I'm still learning...”

Despite the academic challenges, he described the program as:

"Interesting! ...Exciting! ...A good experience...what I was wanting and worthwhile...”

When asked to comment on the content of the program, about the course overall, the preparation needed and the aspect of attending lectures and tutorials and, the Fellow responded:

“... I liked this way of learning... I was able to access the computer easily at the accommodation... I had done a computer course in Vanuatu, but you need to make sure the next students have basic computer skills”.

The comments on clinical were positive, especially those in the primary health care settings.

"Primary health care settings were good, especially Ambulance. I can use this to work on a Memorandum [of Understanding] with the Ambulance Service in Vanuatu and to train the workers... I learnt about drugs, a big 
help. Our drugs were limited...We don't have much trouble with withdrawal. We don't have any policies about this in Vanuatu... we need to develop some health promotion programs... Vanuatu police are developing a health promotion program... so we will be working with them on this... I'm taking an article to them"

He found the rural health placement was most relevant to the Vanuatu context:

"It was good to observe private sector... it was OK, but I think it would be better to go to public hospital where they are more unstable and acute cases like... at the Missenden unit [public hospital] and Bloomfield [rural health]... this was very good. The accommodation was excellent at Bloomfield as I made friends there. The nursing was more realistic to the Vanuatu situation.”

The Fellow perceived that a benefit of the mental health program was the opportunity for him to provide future leadership related to regulation of mental health practice upon their return to Vanuatu:

"It gave me so many ideas to try in Vanuatu. I now feel competent in doing Mental Health Assessments now and I hope I can teach nurses about this. I learnt a lot about medications and handling unstable patients. We don't have many medications but I need to put into place some primary health care programs, especially with community education... I will need to work with many departments in the Ministry and hospital and community to help Mental Health develop in my country”.

The Fellow's overall evaluation of the program were very positive, with many suggestions provided as to how to enhance teaching and learning and increased awareness of future collaboration with our next ALE project.

\section{Discussion}

This was the first project and a pilot study that a Mental Health Fellowship had been undertaken in partnership between the $\mathrm{VMOH}$ and the ACU, North Sydney. Preliminary feedback from Fellow and the stakeholders involved in providing the classroom and clinical experiences indicated the program was successful. They were frequently challenged when trying to apply some of the theoretical constructs that were discussed in the mental health theoretical units, due to the lack of infrastructure in Vanuatu ${ }^{[10]}$. For example, when it came to discussions about medications, Vanuatu has an endorsed medication list which is limited, containing few alternatives. The Fellow was able to identify deficits related to health legislation for the management of mental health patients in Vanuatu and he was able to outline the beginnings of processes to address this issue with the Ministry of Health upon his return.

Another significant challenge for the Fellow was related to the academic expectations, particularly with respect to meeting academic writing standards. This issue was initially raised by staff involved in the theoretical unit where they observed the Fellow's lack of academic writing in class ${ }^{[12]}$. However, the academic staff identified that the Fellow's ability to verbally describe his understanding and application of the theory was satisfactory. This disparity most likely reflects the fact that the Ni-van culture is predominantly one of story-telling to ensure that history and 'kastom' ${ }^{[8]}$ is sustained. Whilst the assistance of University support services was offered to help with his written skills, it became apparent as the program progressed and in the evaluation by the Fellow that simply offering access to these services was not effective. A much more structured approach will be necessary for future programs, and access to services such as the International Office and Student Support will need to be scheduled into the program, rather than offered as optional.

The Fellow recommended that attendance at an English bridging course would be helpful prior to coming to Australia. Accordingly, attendance at a bridging course at the University of the South Pacific will be highly recommended for future Fellows, prior to them undertaking a Fellowship. A further recommendation was to ensure that future Fellows possess basic computer skills. This could also be addressed through the University of the South Pacific if required. In addition, it 
was suggested that future Fellowships look to bring at least 2 nurses to Sydney to assist in reducing the loneliness described by this Fellow.

There were also clinical elements of the program identified where improvements were suggested by the Fellow, such as increasing the number of visits to rural placements and Indigenous services as the Fellow found this aspect most relevant to the Vanuatu context. Attendance at a paramedic workshop on suicide prevention workshop was identified by the Fellow as particularly useful and relevant and should be included in future programs. The Fellow identified that it allowed him to identify opportunities to work with law-enforcement and other stakeholders upon his return to develop care pathways, to improve patient care both pre-hospital and in the primary and tertiary health settings. Clinical mentoring in these mental health placements was integral to enhance the learning.

Upon completion of the program, mentors assisted the Fellow to develop a 12 month plan regarding application of his learning to Vanuatu's identified needs in mental health following his return to Vanuatu. The Fellow returned to Vanuatu where he linked with other emerging mental health focal points that were being developed simultaneously through other strategies, to provide leadership and advice with respect to developing local staff and establishing mental health services in key locations. The Fellow returned to his substantive positions at Vila Central Hospital, as the focal point for assessment of all mental health patients who present there.

In follow-up communication since his return to Vanuatu, the Fellow has reported that he has been able to participate in and lead skills-transfer workshops involving his colleagues, and has been instrumental in the promotion of mental health leadership in the region. The skills development has helped raise the profile of mental health and he has become a valuable clinical resource for clinicians and the local communities. The Fellow has described his role in the process of developing a National Mental Health Committee, which will be responsible for looking at mental health service and policy development for the country. Despite this positive feedback, the Fellow has described the challenges of addressing mental health education in nursing community; however he has noted a slow but real change of attitude towards mental health patients and issues amongst nursing leaders.

Whilst in Vanuatu in July 2011, the project team undertook a follow-up visit to evaluate the outcomes of the Fellowship Program. The team member observed the Fellow at work in Vila Central Hospital and interviewed him regarding implementation and progress of mental health policy and practice in addressing the scope of practice and specific needs of the practitioners in local setting. The Fellow indicated that he was responsible for managing all acute mental health admissions to the hospital and the new mental health clinic at Vila Central Hospital. He reported that the he was able to apply learning from the ALA program, not only at the hospital and in newly developing mental health clinics, but in the community outreach program rolled-out in other provinces. He was also part of a team in reviewing the mental health drugs available in Vanuatu and had been asked to lecture in the mental health at the Vanuatu Centre for Nurse Education. To further develop his skills, the Fellow established links with an Australian GP who had developed a mental health outreach program in Vanuatu ${ }^{[4]}$ at that time.

\section{Limitations}

There are a number of limitations related to the Fellowship program between the VMOH and ACU, the greatest perhaps being the fact that the program is dependent on the availability of grant funding, thus impacting sustainability of the program. With respect to mental health service development in Vanuatu, the contribution of one Fellow, despite their very best efforts, has been limited, in the absence of a broader formal clinical network, formal education program, and defined and funded career structure. However, the continued work to establish the National Mental Health Committee and the recent appointment of a National Mental Health Co-ordinator by the $\mathrm{VMoH}$ has meant that the profile of mental health is slowly being raised, and will hopefully lead to a more co-ordinated response to mental health challenges in Vanuatu. 
Upon completion of the theoretical and practical units at ACU the Fellow received a certificate of attainment. However, this certificate will not be recognized as a formal qualification. At best, should the Fellow wish to pursue a formal qualification, the certificate only assumes testimony to the fact that he has an interest in mental health, and completed some foundation units related to mental health. Currently, to achieve a formally recognised mental health qualification, like so many other post-graduate qualifications, Ni-Vanuatu nurses need to leave their country.

In order for Vanuatu to be able to offer a post-graduate qualification in mental health, they will require significant re-structuring of nurse education to increase the basic qualification from certificate to diploma level. Whilst Fiji appears to have achieved this, and offers a post-graduate mental health qualification ${ }^{[3]}$ they have had a head start of almost a decade on Vanuatu. Vanuatu is fortunate in that there is increasing outside interest in assisting them in the development of mental health services. However, one of the noted limitations is the lack of clearly co-ordinated efforts which continue to create a piecemeal approach to service development, and on occasion, duplication of effort.

\section{Conclusions}

The nurse who completed the Fellowship program is recognised as an emerging leader in the field of mental health in Vanuatu. With continued support, it is expected that he will undertake training of other nurses, nurse-aides and village health-workers, facilitating education to communities regarding inclusion of mental health assessment and management as a routine part of primary health care. Whilst ACU is presently involved it is envisaged that sometime in the not too distant future, the Vanuatu School of Nurse Education will take over this role, similar to what occurred with the mental health nursing postgraduate program in Fiji ${ }^{[12]}$.

\section{I mplication}

In order to establish robust and sustainable mental health services, in the long term, the Government of Vanuatu will need to review the current nurse education program, funding and career structure, to ensure that there are positions for qualified staff to take up. In the interim, better co-ordination of the multiple strategies and investments in mental health is required to ensure maximum value is realised.

\section{Acknowledgements}

The authors acknowledge the Australian Government Australian Leadership Awards Fellowships for their contribution and support for this study.

\section{References}

[1] The Vanuatu Mental Health Policy and Plan 2009-2015. The Ministry of Health Government of Vanuatu: Vanuatu Government Publication. 2009.

[2] Andrews, M. \& Roberts, D. Supporting student nurses learning in and through clinical practice: the role of the clinical guide. Nurse Education Today. 2003; 23: 474-481. http://dx.doi.org/10.1016/S0260-6917(03)00076-5

[3] Vanuatu National Statistics Office (VNSO). Vanuatu Census Report www.vnso.gov.vu. 2009.

[4] The Vanuatu Mental Health Training Action 2009 - 2013. Vanuatu Draft Health-Sector Policy, 2008.

[5] WHO. Situational analysis of mental health needs and resources in Pacific Island countries. Centre for Mental Health Research, Policy \& Service Development World Health Organisation: January 2005; 116-118.

[6] New Zealand AID (NZAID). A new paradigm for funding social enterprises. Connections. June 2009; (15): 8-11.

[7] Benson, J., Pond, D., Funk, M., Wang, X. \& Tarivonda, L. A new era in mental health care in Vanuatu. International Journal of Family Medicine.2011; ID 590492 Doi:101155/2011/590492.

[8] Kaloris, P. Key Psychosocial \& Legislative Issues For Carers of People with Mental Disorder/Illnesses in Vanuatu. Presented at the Foundation for the People of the South Pacific Vanuatu at the Antares Foundation Inc. November 2009. 
[9] Kuruvilla, G. Vanuatu: happiest nation on earth, mental health and the Church. Australasian Psychiatry. 2010; 18(1): 63-65. PMid:20136535 http://dx.doi.org/10.3109/10398560903298752

[10] Hughes, F. Mental Health in the Pacific: the role of the Pacific Island Mental Health Network (PIMHNET) Pacific Health Dialog. 2009; 15(1). PMid:19585749

[11] Foster K., Usher K., Gadai S. \& Taukei R. 'There is no health without mental health': implementing the first mental health nursing program in Fiji. Contemporary Nurse. 2009; 32(1-2): 179-186. PMid:19697988 http://dx.doi.org/10.5172/conu.32.1-2.179

[12] Funk, M., Drew, N., Freeman, M. \& Faydi, E. Mental health and development: Targeting people with mental health conditions as a Vulnerable group, WHO 2010.

[13] Geertz, C. After the Fact: Two Countries, Four Decades, One Anthropologist. The Jerusalem-Harvard Lectures. Cambridge and London: Harvard University Press; 1995.

[14] Flyvbjerg, B. Five Misunderstandings about Case-Study Research. Qualitative Inquiry. 2006; 12: 219-245. http://dx.doi.org/10.1177/1077800405284363

[15] Rose, M. \& Best, D. Transforming practice through clinical education, professional supervision and mentoring. Sydney: Elsevier; 2005.

[16] Henderson G., Estroff S., Churchill L., King N., Oberlander J., et al. editors. Social and cultural contributions to health, difference, and inequality. Volume II: The social medicine reader 2nd edition Durham: Duke University Press.2005.

[17] Patton, M. Two Decades of Developments in Qualitative Inquiry: A Personal, Experiential Perspective. Qualitative Social Work. 2002; 1: 261. http://dx.doi.org/10.1177/1473325002001003636

[18] Usher, K., Rabuka, I., Nadakuitavuki, R., Tollefson, J. \& Luck, L. Nursing and the development of nursing education in Fiji. Australian Journal of Advanced Nursing. 2004; 21(3): 28-31.

[19] WHO. The effectiveness of mental health services in primary care: The view from the developing world. WHO: Geneva.2001.

[20] Vanuatu www.who.int?mental_health policy/pimhnet/Pacific_islands_needs_assessment 